\title{
Die kutane Tuberkulinimpfung nach v. Pirquet.
}

800 Fälle aus der Universitätskinderklinik Freiburg i. Brsg. (Direktion: Professor Salge.)

Von

\section{H. Berberich.}

Die Schwierigkeit der Diagnose der Tuberkulose besonders im Kindesalter ist den Tuberkuloseforsehern schon seit langem ein steter Stachel zur Erfindung eines sicheren, einwandfreien Verfahrens. Die probatorische, subkutane Tuberkulinimpfung nach $\mathrm{Koch}$ wurde von den meisten Ärzten abgelehnt, da sie teils zu gefährlich ist wegen der folgenden eventuellen Allgemeinerscheinungen, teils unausführbar, da die Vorbedingung der Fieberfreiheit meistens nicht erfüllt ist. Als dann v. Pirquet sein neues Verfahren der kutanen Impfung mit K och s Alttuberkulin im Mai 1907 in der Berliner medizinischen Gesellschaft vortrug, das weder die Fieberfreiheit voraussetzte noch die Gefahr von Allgemeinersscheinungen hatte, da dauerte es nicht lange, bis dieses diagnostische Hilfsmittel sich die meisten Kliniken und das Vertrauen vieler Ärzte eroberte. Besonders die Kinderärzte und -Kliniken nahmen diese neue Methode mit Freuden auf, die ja die aktive Tuberkulose der ersten Lebensjahre, namentlich des ersten Jahres, mit Sicherheit anzeigen sollte, da die inaktive erst nach dem zweiten und dritten Lebensjahre auftritt.

Im Juli 1907 berichtete dann v. Pirquet über Impfungen an 360 Kindern vom Säuglingsalter bis zu 14 Jahren. Von diesen 360 Kindern reagierten 124 positiv, ansteigend von $0 \%$ in den ersten 4 Lebensmonaten bis zu 60\% im Alter von 8-14 Jahren. Darunter waren 294 klinisch nicht sicher tuberkulös, von welchen 56 positiv reagiert hatten. Und unter diesen klinisch nicht sicher Tuberkulüsen 
bezeichnet er 261 als klinisch nicht tuberkuloseverdächtig, bei denen die Reaktion $32 \mathrm{mal}$ positiv war, ansteigend von $0 \%$ vom Alter von 0-4 Monaten bis zu 35\% im Alter von 8-14 Jahren.

v. Pirquet erwähnte schon bei dieser Arbeit, dass das Versagen der Impfung bei Kachektischen und schwer Tuberkulösen (Miliartuberkulose, Meningitis tuberculosa) seine Analogie in der Unempfindlichkeit dieser Kranken gegen die Tuberkulininjektion findet, von der in den letzten Lebenstagen grosse Dosen ohne Wirkung vertragen werden und erklärt: „Wir können von der Impfung prinzipiell nicht mehr erwarten als von der Injektion, sie stellt nur eine wesentlich bequemere und ungefährlichere Methode dar“. Im September 1907 veröffentlichte er die Arbeit: „Der diagnostische Wert der kutanen Tuberkulinreaktion bei der Tuberkulose des Kindesalters auf Grund von 100 Sektionen." Hierbei fand er, dass von 100 Fällen 52 ohne tuberkulöse Veränderungen waren, die auch negativ reagiert hatten. Von den übrigen 48 hatten $310 \%$ reagiert. Bei 34 Fällen mit tötlicher Tuberkulose war 21 mal positiver, $13 \mathrm{mal}$ negativer Ausfall der Reaktion; allerdings waren 24 Fälle erst in den letzten 10 Lebenstagen geimpft worden und unter diesen waren die 13 Fälle mit tödlicher Tuberkulose, die negativ reagiert hatten. 13 Fälle ergaben als Nebenbefund der Sektion Tuberkulose, ron diesen hatten 9 positiv, 4 negativ reagiert. Nur in einem Fall konnte bei der Sektion keine tuberkulöse Veränderung gefunden werden, während die Reaktion positiv gewesen war.

An diese ersten Veröffentlichungen von v. Pirquet selbst schliesst sich eine grosse Reihe von Arbeiten an, die den Zweck hatten, die Sicherheit und den Wert der Pirquetschen Reaktion zu prüfen. So berichtet im Oktober $1907 \mathrm{Lenhartz}$ über seine Erfahrungen mit der Calmetteschen Ophthalmoreaktion und der Pirquetschen kutanen Tuberkulinprote. Bei 110 Personen war die Pirquetsche Probe gemacht worden und die Reaktion war 22 mal positiv unter 37 klinisch sicher Tuberkulösen. Die übrigen 15 waren schon vorher mit Tuberkulin behandelt; von ihnen war 6 mal negative Reaktion, 9 mal positive (darunter 4 angedeutat, 3 fraglich, 2 deutlich).

10 klinisch Tuberkulosefreie (9 Erwachsene) gaben 5 mal positive, 5 mal negative Reaktion.

63 auf Tuberkulose Verdächtige gaben

23 mal positiven Pirquet (darunter 19 Erwachsene),

40 mal negativen Pirquet (darunter 26 Erwachsene).

Über 344 Fälle aus der Heidelberger Kinderklinik berichtet Feer im Januar 1908. Von diesen hatten 
65 positiven Pirquet $(=19 \%)$.

Schon bei diesen verhältnismässig kleinen Zahlen konnte er eine regelmässige Steigerung des Prozentsatzes der Positiven mit zunehmendem Alter nachweisen, wie dies durch Sektionsergebnisse und spätere Statistiken aus anderen Städten bestätigt wurde, worauf auch schon v. Pirquet aufmerksam gemacht hatte.

Die positiven Fälle stiegen von $0 \%$ im ersten Lebenssemester bis auf $57 \%$ zwischen 10 und 15 Jahren. Von 112 Säuglingen (0-12 Monate) reagierten nur 3 positiv mit nachheriger Bestätigung der Diagnose durch die Sektion. Die 344 Fälle teilte er ein in

1. Klinisch sicher Tuberkulöse:

25 Fälle mit 24 mal positivem Pirquet. Der 25. Fall war ein sehr elendes, 13 Monate altes Mädchen mit Peritonitis tuberculosa.

2. Tuberkuloseverdächtige :

28 Fälle: positiv reagierten $14(==50 \%$ ).

3. Klinisch Tuberkuloseunverdächtige:

291 Fälle: 27 positive Fälle $(=90 \%)$.

Auch hier das Steigen mit zunehmendem Alter von $0 \%$ im ersten Halbjahr bis $35_{0}^{0}$ zwischen 10 und 15 Jahren. Unter den 344 Geimpften waren 50 seitens direkter Verwandter belastet, von denen 19 positiv reagierten.

Einen wertvollen Beitrag zu den Ergebnissen mit der Pir $q$ ciet schen Reaktion bei Kindern lieferte Morgen roth in seiner Arbeit über: „Die kutane Tuberkulinimpfung nach v. Pirquet bei Kindern des ersten Lebensjahres", die im Juni 1908 erschien.

In der Kinderklinik der Akademie für praktische Medizin zu Köln wurde schon damals die vereinfachte Impfung mit unverdünntem Alttuberkulin nach $\mathrm{K}$ o $\mathrm{ch}$ angewandt, wie dies wohl seitdem allgemein geschieht. Unter 200 Fällen reagierten 10 positiv $(=5 \%)$. Die Sektionsergebnisse stimmten mit der Reaktion überein.

Je mehr man durch diese und andere Arbeiten den Wert der Pirquetschen Reaktion für die Diagnose der kindlichen Tuberkulose schätzen lernte, um so mehr Material wurde in den nächsten Jahren publiziert. Aus der grossen Fülle dieser Arbeiten seien hier nur: einige herausgegriffen, die aus geographisch weit auseinander liegenden Gegenden stammen, da es doch interessant ist, derartige Statistiken aus den verschiedensten Gegenden miteinander zu vergleichen, die einerseits uns die Übereinstimmung der Zuverlässigkeit der Pirquetschen Reaktion deutlich vor Augen führen, andererseits aber auch uns Aufschluss über die verschiedenartige Verbrei- 
tung der Tuberkulose in den verschiedenen Gegenden Deutschlands und Österreichs geben.

Veit berichtet 1909 in seiner Arbeit aus dem Allgemeinen Krankenhause Hamburg - Eppendorf über 550 Impfungen bei Kindern. Darunter waren 282 Säuglinge, von denen 10 positiv reagierten $(=5,8 \%)$ ansteigend von $0 \%$ im ersten Monat bis zu $33,3 \%$ im 12. Monat. Alle 10 sind tuberkulös belastet, 4 davon starben. Die Diagnose, gestützt auf den positiven Ausfall der Pirquetschen Reaktion, wurde durch die Sektion bestätigt! Ausser den Säug. lingen wurden noch 368 Kinder bis zum 14. Lebensjahr mit Puberkulin geimpft.

114 reagierten positiv, ansteigend von $5,7 \%$ im 1 . Lebensjahre bis $46,6 \%$ im 14. Jahre. 254 reagierten negativ. 29 waren klinisch sicher tuberkulös, von welchen 27 positiv, 2 negativ reagiert hatten. $\mathrm{V}$ e it schliesst daraus, dass alle Lungentuberkulosen des Kindesalters, die sich nicht im Endstadium befinden, eine positive Reaktion zeigen.

39 Fälle waren auf Tuberkulose verdächtig,

33 reagierten positiv, 6 negativ.

Unter diesen 6 waren 2 Fälle, bei denen die Röntgenaufnahmø den Verdacht auf Tuberkulose bestätigte, während der negative Ausfall der Pirquetschen Reaktion nicht verwertet werden durfte, da beide Kinder sich in sehr schlechtem Allgemeinzustand befanden.

Die positiven Fälle bespricht er nicht weiter; da ja der Ausfall der Reaktion bei Kindern über 1 Jahr nicht entscheidend sei. Je jünger allerdings ein Kind, um so mehr sei auf die Reaktion zu geben.

Interessant war der jeweilige Sektionsbefund. Von 118 Todesfällen kamen 116 zur Autopsie (2 Sektionen verweigert).

1. 78 waren klinisch nicht tuberkulös, hatten negativ reagiert und boten auch bei der Sektion keinen tuberkulösen Befund.

2. Sektionen Tuberkulöser: 38 Fälle, von denen 16 positiv, 22 negativ reagiert hatten.

19 Fälle waren an miliarer Tuberkulose zugrunde gegangen, von denen 9 positiv reagiert hatten. Der negative Ausfall bei den restierenden 10 ist leicht erklärt durch die Widerstandsunfähigkeit des Körpers, im Endstadium der Tuberkulose auf Tuberkulin zu reagieren (Prognose immer ad malum vergens bei klinisch schwerer Tuberkulose mit negativem Pirquet). Unter den 19 waren 9 Fälle von Meningitis tuberculosa, von denen 3 noch positiv reagiert hatten. Veit fiel es bei den Sektionen auf, „dass bei milianer Aussaat und nur geringfügigen, älteren tuberkulösen Veränderungen, die gar 
keine Krankheitserscheinungen hervorgerufen hatten, die Kutanreaktion positiv verlaufen ist, während bei vorher bestehender $\mathrm{Er}$ krankung an Tuberkulose und grösseren Herden mit Kavernenbildungen die Kutanreaktion negativ verlaufen war".

Seine Ergebnisse an den Erwachsenen interessieren uns hier nicht, da man ja heute weiss, dass die Pirquetsche Reaktion bei Erwachsenen diagnostisch nicht verwertet werden darf.

Kreisarzt Hillenberg hatte sich 1909 die Mühe genommen, die Einwohner der ungefähr 3000 Köpfe zählenden Stadt Zille durchzuimpfen. Leider fängt seine Arbeit erst bei den Schulkindern von 5 Jahren an, während die für diese Arbeit besonders interessierenden ersten Lebensjahre nicht in Betracht gezogen wurden.

Einiges Interesse aber bieten folgende Zahlen bei Kindern, die eben in die Schule kamen, bzw. die Befunde nach dem ersten Schuljahr, die uns doch wertvollen Aufschluss über die Zeit der Entstehung der Tuberkulose geben. Es waren:

\begin{tabular}{c|c|c|c}
\hline \hline Alter & $\begin{array}{c}\text { Zahl } \\
\text { der Kinder }\end{array}$ & $\begin{array}{r}\text { positive } \\
\text { Reaktion }\end{array}$ & Prozent \\
\hline $\begin{array}{l}5-6 \text { Jahre } \\
6-7 \pi\end{array}$ & 27 & 1 & $\begin{array}{r}4,54 \\
78\end{array}$ \\
& 20 & 25,96
\end{tabular}

Darunter waren neu eingeschult:

\begin{tabular}{l|l|l|l|l}
$5-6$ & Jahre & 27 & 1 & 4,54 \\
$6-7$ & 47 & 5 & 8,39,
\end{tabular}

während unter den übrigen 31 Kindern im Alter von 6-7 Jahren nach dem ersten Schuljahre 11 positiv reagierten, das sind $38,71 \%$.

Ob man allerdings aus diesen kleinen Zahlen allgemeine Schlüsse ziehen darf, besonders, da in Zille fast keine Industrie besteht, möchte ich bezweifeln.

An einem grossen Material behandelte $\mathrm{Hamburger}$ in Wien in seiner Arbeit über: „Allgemeine Pathologie und Diagnostik der Kindertuberkulose" (1910) auch die Tuberkulosehäufigkeit. Angeregt wurde er durch den überraschenden Befund $\mathrm{Na} \in \mathrm{gelis}$, der in $97 \%$ der in Zürich verstorbenen Erwachsenen Zeichen von Tuberkulose fand. $\mathrm{Hamburger}$ fand dann bei 848 Kinderleichen für den Zeitraum von $7-10$ Jahren $63 \%$, für das Alter von 11-14 Jahren 70\% Tuberkulose. (Für die Zeit unter 7 Jahren führt er die Zahlen nicht an.) Er unterwarf dann 509 Fälle des St. Anna-Kinderhospitals in Wien, die nicht wegen 
einer tuberkulösen Krankheit eingeliefert waren, der Tuberkulinprobe zuerst nach von Pirquet, während er den noch Negativen intrakutan Tuberkulin injizierte (Stichreaktion nach Escherich). Die auch auf die Stichreaktion nicht reagierenden bezeichnet er als negativ.

Die nun folgenden Zahlen dürfen nicht für die Statistik der kutanen Tuberkulinprobe nach v. Pirquet verallgemeinert werden, da auch der positive Ausfall der intrakutanen Injektion verwertet wurde. Von den 509 Kindern reagierten $271=53 \%$ auf Tuberkulin. Leider sind darunter nur 23 Fälle des 1. Lebensjabres, die er selbst als nicht verwertbar bezeichnet, da die Zahl zu klein ist, um für allgemeine Schlüsse verwendet werden zu können. Von diesen hatte allerdings kein einziger positiv reagiert. Bei den übrigen stieg die Zahl der positiv Reagierenden von $9 \%$ im 2. Lebensjahre bis zu $94 \%$ im 14. Lebensjahre. Seine Zahlen beziehen sich nur auf die ärmere Bevölkerung Wiens, die aber mit den von Ganghofer in Prag gefundenen Zahlen ziemlich übereinstimmen, die von $8,5 \%$ im 1 . Jahre, $12 \%$ im 2. Jahre bis auf $80 \%$ im 13. und 14. Jahre steigen. $\mathrm{Ha} \mathrm{mburg}$ er zieht daraus den Schluss, dass diese Zahlen wohl für die ärmere Bevölkerung Mitteleuropas gelten dürfen!

Feer berichtet 1910 über 2000 Fälle, die in der Heidelberger Universitätskinderklinik der Kutanreaktion nach v. Pir qu e t unterworfen wurden. (Siehe auch Doktordissertation von Lotte Behrend: „Über den Wert der Pirquetschen Kutanreaktion bei Kindern." 2000 Fälle aus der Heidelberger Universitätsklinik.)

Von diesen 2000 Fällen hatten 452 positiv reagiert $=23 \%$ (ansteigend von $1,7 \%$ im 1 . Lebensjahre bis zu $53 \%$ im Alter von 10-14 Jahren).

171 Kinder waren klinisch sicher tuberkulös $(=8,5 \%)$, von denen 163 positiv reagierten $=95 \%$ (75\% im ersten Halbjahr, dann immer 95-100\%).

Tuberkuloseverdächtig waren $97=5 \%$, von denen 62 positiv reagierter $=64 \%$ (steigend von $50 \%$ im ersten Lebensjabre bis auf $94 \%$ im Alter von $10-14$ Jahren).

Von den 1732 klinisch tuberkulosefreien reagierten nur

277 positiv $=13 \%$, ansteigend von $0 \%$ im ersten Lebenssemester bis auf $38 \%$ im Alter von 10-14 Jahren.

120 Fälle kamen zur Sektion. Dabei konnte bei 24 Fällen mit positiver Reaktion nur 2 mal kein tuberkulöser Herd gefunden werden. 
Auch hier wieder die Tatsache der Spezifität der P ir q u et schen Reaktion für Tuberkulose. Alle Fälle von Tuberkulose - aktive und inaktive - reagierten mit Ausnahme der Fälle von sehr fortgeschrittener und miliarer Tuberkulose und allgemeiner Kachexie.

Wertvoll hält $\mathrm{Fe}$ er die Probe in den ersten 3 Lebensjahren, weil sie fast stets eine aktive Tuberkulose anzeigt. Jenseits dieser Zeit ist der negative Ausfall bei klinisch sicherer Tuberkulose mehr ad malum vergens. Schlimme Folgen der Pirquetschen Reaktion wurden nie beobachtet, nur manchmal hochgradige Blasenbildung bei Skrofulose.

Im Juniheft 1911 der Zeitschrift für Kinderheilkunde berichtet Radziejewski über 366 Sektionen (39 aus der Breslauer Universitätskinderklinik, 327 aus der Wiener Universitätskinderklinik und dem K. K. Franz-Josefspital). 153 hatten positive Kutanreaktion gehabt, ansteigend von $5,4 \%$ im ersten Vierteljahr bis zu $100 \%$ im Alter von 10-14 Jahren.

Bei $184=$ ca. $50 \%$ wurde bei der Obduktion keine Tuberkulose gefunden; von ihnen hatten 4 positiv reagiert. Eine genauere mikroskopische Untersuchung auf Tuberkulose oder gar Tierversuche waren bei diesen 4 Fällen nicht gemacht worden.

182 hatten bei der Sektion tuberkulöse Befunde gezeigt, von denen 149 positiv reagiert hatten. Die Tuberkulosehäufigkeit steigt hierbei von 5,4\% im ersten Vierteljahr bis zu $100 \%$ im Alter von 10-14 Jahren.

Die hier angeführten Berichte über die Pirquetsche Reaktion zeigen eine Verschiedenheit der Tuberkulosehäufigkeit in verschiedenen Gegenden. Es erscheint daher interessant, die Ergebnisse der Reaktion in einer mittelgrossen Stadt, wie es Freiburg ist, zu betrachten, wo einerseits Orts- und soziale Verhältnisse günstig sind, während andererseits die Thberkulose dort eine sehr verbreitete Volkskrankheit ist. Ich halte mich dabei an das Material der Freiburger Kinderklinik, in der seit einiger Zeit jedes Kind der Pirquetschen Reaktion unterzogen wird.

Das Material der hiesigen Kinderklinik besteht grösstenteils aus Kindern der unteren Volksschichten, so dass sich die Zahlen, über die ich berichte, etwas höher stellen werden, als wenn ich auch das Material der in besseren äusseren Verhältnissen Iebenden Kindern mit in Berechnung hätte ziehen können. Da sich aber unter den 800 Fällen, die seit 1909 geimpft wurden, nur 40 Fälle (5\% aller Fälle) mit klinisch sicherer Tuberkulose befinden und 
andererseits eine grosse Anzahl von Kindern wegen leichter Affektionen (Hautkrankheiten usw.) aufgenommen waren, so glaube ich, dass dieser Bericht als ziemlich objektiv angesehen werden darf und dass diese Zahlen mit denen mittelgrosser Städte Deutschlands (ca. 100000 Einwohner) ziemlich übereinstimmen werden.

Im Gegensatz zu anderen Berichten teile ich dieses Material nur in

1. klinisch sicher tuberkulöse und

2. klinisch nicht sicher tuberkulöse Fälle ein, während ich den Verdacht auf Tuberkulose für einen zu subjektiven Begriff halte, als dass man die entsprechenden Zahlen für vollwertig halten dürfte. Ich versuchte nun, diese Zahlen noch objektiver zu gestalten, indem ich 111 zurzeit völlig gesunde Kinder durchimpfte, von denen sich 40 Kinder im Alter von 4-14 Jahren in den Monaten Juni und Juli 1911 im hiesigen städtischen Solbad befanden. die übrigen $71 \mathrm{im}$ Alter von 1-8 Jahren (darunter 42 im Alter von 1-4 Jahren) in der städtischen Krippe verpflegt wurden. (Diese Zahlen fügte ich zu denen der Klinik hinzu; sie selbständig zu verwerten, dürfte nicht berechtigt sein, da sie zu klein sind für allgemeine Schlüsse.)

Unter den 800 Kindern zeigten

513 negative Reaktion,

287 positive Reaktion $=36 \%$.

Diese verteilen sich auf die verschiedenen Lebensjahre folgendermassen :

\begin{tabular}{|c|c|c|c|c|}
\hline \multicolumn{2}{|c|}{ Alter } & $\begin{array}{l}\text { Anzahl } \\
\text { der Fälle }\end{array}$ & $\begin{array}{l}\text { Positive } \\
\text { Reaktion }\end{array}$ & $\begin{array}{c}\text { Positive } \\
\text { Reaktion } \\
\text { in Prozent- } \\
\text { zahlen }\end{array}$ \\
\hline \multicolumn{2}{|c|}{$0-6$ Monat } & 156 & 16 & 10,3 \\
\hline \multirow{2}{*}{\multicolumn{2}{|c|}{$\begin{array}{l}6-12 \\
1-2 \text { Jahre }\end{array}$}} & 57 & 14 & 24,6 \\
\hline & & 137 & 35 & 25,5 \\
\hline \multicolumn{2}{|c|}{$2-3$} & 94 & 26 & 27,6 \\
\hline \multirow{2}{*}{$\begin{array}{l}3-4 \\
4-5\end{array}$} & , & 61 & 27 & 44,2 \\
\hline & $n$ & 51 & 29 & 56,8 \\
\hline $5-6$ & $"$ & 45 & 26 & 57,7 \\
\hline $6-8$ & , & 56 & 31 & 55,4 \\
\hline $8-10$ & $\pi$ & 70 & 40 & 57,0 \\
\hline \multirow[t]{2}{*}{$10-15$} & n & 73 & 43 & 58,8 \\
\hline & & 800 & 287 & 35,9 \\
\hline
\end{tabular}


Es ist also eine deutliche Zunahme der Häufigkeit der positiven Reaktion zu konstatieren, wobei auf den Unterschied, ob inaktive oder aktive Tuberkulose vorliegt, nicht Rücksicht genommen wurde. Betrachten wir nun die 40 klinisch als sicher tuberkulös diagnostizierten Fälle

\begin{tabular}{|c|c|c|c|c|c|}
\hline \multicolumn{2}{|c|}{ Alter } & $\begin{array}{c}\text { Anzahl der } \\
\text { klinisch } \\
\text { sicher } \\
\text { Tuberkulösen }\end{array}$ & $\begin{array}{c}\text { Prozentsatz im } \\
\text { Verhaltnis zur } \\
\text { Gesamtzahl der } \\
\text { Fälle }\end{array}$ & $\begin{array}{r}\text { Positive } \\
\text { Reaktion }\end{array}$ & $\begin{array}{l}\text { Prozentsatz der } \\
\text { positiv. Reaktion } \\
\text { d. klinisch sicher } \\
\text { Tuberkulösen }\end{array}$ \\
\hline \multirow{3}{*}{\multicolumn{2}{|c|}{$\begin{array}{l}0-6 \text { Monat } \\
6-12 \\
1-2 \text { Jahre }\end{array}$}} & 3 & 1,9 & 2 & 66,6 \\
\hline & & 2 & 3,5 & 2 & 100 \\
\hline & & 7 & 5,1 & 5 & 71 \\
\hline $2-3$ & $\pi$ & 4 & 4,2 & 4 & 100 \\
\hline $3-4$ & , & 5 & 8,2 & 3 & 60 \\
\hline $4-5$ & . & 4 & 7,8 & 2 & 50 \\
\hline $5-6$ & , & 2 & 4,4 & 1 & 50 \\
\hline $6-8$ & n & 3 & 5,4 & 2 & 66,6 \\
\hline $8-10$ & $n$ & 3 & 1,3 & 3 & 100 \\
\hline $10-15$ & $\pi$ & 7 & 9,6 & 7 & 100 \\
\hline
\end{tabular}

so können wir hier zwar keine regelmässige Steigerung mit den Lebensjahren konstatieren (schon wegen der kleinen Zahlen lässt sich nichts Bestimmtes aus diesem Befund schliessen), aber wir finden doch, dass bei aktiver Tuberkulose die Reaktion neistens positiv ausfällt. Die Prozentzahlen der positiven Reaktion bei diesen Fällen halten sich daher auch dauernd über $50 \%$. Von dissen 40 Tuberkulösen hatten 9 negativen Pirquet; nämlich die 8 Fälle, die später unter den Sektionsberichten von sicherer Tuberkuloso bei negativem Pirquet genauer erwähnt werden, und folgender Fall von Miliartuberkulose.

E. H., $4^{3} / 4$ Jahre altes Mädchen.

Klinische Diagnose: Miliartuberkulose. Allgemeinzustand sehr schlecht. Puls kaum zu fühlen. Über beiden Lungen zerstreut mittelblasiges Rasseln. Pirquet 16 Tage ante exitum negativ.

Sektion verweigert.

Unter den 760 klinisch nicht sicher Tuberkulösen hatten 260 positive Reaktion $=34,2 \%$ und zwar: 


\begin{tabular}{|c|c|c|c|}
\hline Alter & $\begin{array}{c}\text { Anzahl } \\
\text { der Fälle }\end{array}$ & $\begin{array}{l}\text { Positive } \\
\text { Reaktion }\end{array}$ & $\begin{array}{l}\text { Prozente } \\
\text { derselben }\end{array}$ \\
\hline $0-6$ Monat & 153 & 14 & 9,2 \\
\hline $6-12$ & 55 & 12 & 21,8 \\
\hline 1-2 Jabre & 130 & 30 & 23.0 \\
\hline $2-3$ & 90 & 26 & 28,8 \\
\hline $3-4$ & 56 & 24 & 42,8 \\
\hline $4-5$ & 47 & 27 & 57,5 \\
\hline $5-6$ & 43 & 25 & 58,1 \\
\hline $6-8$ & 53 & 29 & 54,7 \\
\hline $8-10$ & 67 & 37 & 55,2 \\
\hline $10-12$ & 66 & 36 & 54,6 \\
\hline Summe: & 760 & $\overline{860}$ & $34, \div$ \\
\hline
\end{tabular}

Diese Aufstellung zeigt deutlich die Zunahme der inaktiven Tuberkulose mit den Jahren, besonders nach dem 3. Lebensjahre, die vom 4. Lebensjahre an sich dauernd über $50 \%$ hält.

Da ich den Tuberkuloseverdacht nicht verwerte, so finde ich schon im Säuglingsalter (und in den ersten $1-3$ Jahren) einen etwas höheren Prozentsatz der inaktiven Tuberkulose, der sich aber im Vergleich zu den Prozentzahlen bei aktiver Tuberkulose als so klein erweist, dass die Tatsache der Seltenheit der inaktiven Tuberkulose im Säuglingsalter auch für hiesige Verhältnisse gilt. Ein positiver Ausfall der Pirquetschen Reaktion im Säuglingsalter ist also in allen Fällen als wichtiges diagnostisches Hilfsmittel der aktiven Tuberkulose anzusehen.

\section{Sektionen.}

Interessant sind die Ergebnisse der Sektionen. Von 92 Todesfällen kamen 65 zur Sektion, die übrigen 27 wurden leider verweigert. 40 mal wurde bei der Sektion keine Tuberkulose gefunden.

Bei 34 Fällen war auch die Pirquetsche Reaktion negativ gewesen, bei den übrigen 6 Fällen hingegen positiv. Bei einer äusserst peinlichen mikroskopischen Untersuchung bis auf die kleinsten Lymphdrüsen oder bei Tierversuchen wäre vielleicht auch bei diesen Fällen der Nachweis von Tuberkulose gelungen.

1. K. B., 12 Jahre alter Knabe. Klinische Diagnose: Hirnabszess. Von väterlicher und grossmütterlicher Seite tuberkulös belastet. Pirquet positiv.

2. J. D., 9 Monate altes Mädchen. Rachitis, Pneumonie (mit bronchitischen Geräuschen über der linken Lunge). Ergab bei der Aufnabme schwach positiv Pirquet, nach 3 Monaten war bei der Wiederimpfung Pirquet negativ. 
3. L. Sp., 9 Monate altes Mädchen. Bronchitis. Latente Tetanie, Pirquet negativ. Im Alter von 10 Monaten wieder in der Klinik wegen Ernährungsstörungen. Pirquet positiv.

4. J. S., 6 Jahre altes Mädchen. Sepsis. Pirquet positiv.

5. F. M., 53/4 Jahre alter Knabe. Nephritis bei Diphtherie. Morbilli. Bronchitis. Bronchopneumonie. Pirquet positiv.

6. E. T., 13 Monate altes Mädchen. Ernährungsstörung. Bronchopneumonie. Pirquet positiv.

(3 Kinder hatten tuberkulöse Verwandte.)

25 Sektionen ergaben Tuberkulose als Todesursache oder Nebenbefund, von denen 5 tuberkulös belastet waren. Bei 17 Fällen hatte der positive Ausfall der Reaktion die Diagnose unterstützt.

Bei den übrigen 8 Fällen hingegen war die Pirquetsche Reaktion negativ.

1. M. R., 31/2 Jahre altes Mädchen.

Klinisehe Diagnose: Meningitis tuberculosa. Otitis media beiderseits. Pirquet negativ, 1 Tag ante exitum gemacht.

Sektionsdiagnose: Leptomeningitis tuberc. basilaris. Otitis media tuberc beiderseits. Verkalkter tuberkulöser Herd im rechten Oherlappen, miliare Tuberkulose beider Oberlappen. Alte kreidige Tuberkulose der mediastinalen und trachealen, frische Tuberkulose der bronchialen Lymphdrüsen.

Mikroskopisch: auch miliare Tuberkulose der Milz und Leber.

2. A. R., $5 \frac{3}{4}$ Jahre alter Knabe.

Klinische Diagnose: Meningitis tuberculosa. Klinischer Befund: 5 Tage nach der Aufnahme auf der linken Seite trockenes Rasseln. Am 6. Tag (Tag des Exitus) auch Tuberkelbazillen im Iumbalpunktat. Pirquet 5 Tage ante exitum negativ.

Sektionsbefund: Verkalkter Herd in der rechten Lungenspitze. Verkäste Hiluslymphknoten. Miliare Tuberkulose der Lungen. Schleimig-eitrige Bronchitis. Verwachsungen der linken Pleura. Miliare Tuberkulose der Leber und Nieren. Tuberkulöse Basilarmeningitis. Tuberkulose des Plexus chorioideus. Hydrocephalus internus und externus. Tuberkulose der Rückenmarkshäute. zustand.

3. G. G., $13 / 4$ Jahre alter Knabe in schlechtem Ernährungs-

Klinische Diagnose : Chronische Ernährungsstörung. Tuberkulose der Bronchialdrüsen. Pirquet 12 Tage ante exitum negativ.

Sektionsbefund: Tuberkulose der peribronchialen Lymphknoten.

4. H. R., $4 \frac{1}{2}$ Jahre alter Knabe in schlechtem Ernährungszustand. Vater und Mutter lungenkrank. 
Klinische Diagnose: Meningitis tuberculosa. Im Lumbalpunktat Tuberkelbazillen. Pirquet 4 Tage ante exitum negativ.

Sektionsbefund: Kleine Herde verkäster peribronchialer Tuberkulose in beiden Lungen. Tuberkulose der trachealen Lymphknoten. Käsige und frische Tuberkulose der Hiluslymphknoten. Miliare Tuberkulose der Milz und der Leber. Meningitis tuberculosa der Hirnbasis. Geringer Hydrocephalus internus.

5. M. J., $3 \frac{1}{2}$ Jahre altes Mädchen.

Klinische Diagnose: Meningitis tuberculosa. Lumbalpunktion: Tuberkelbazillen negativ. Pirquet 2 Tage ante exitum negativ.

Sektionsbefund: Tuberkulöse Meningitis, besonders der Hirnbasis. Zahlreiche Tuberkeln des Ependyms und der weichen Hirnhäute. Disseminierte Tuberkulose der Lungen. Ältere, zum Teil verhärtete Tuberkel des rechten Oberlappens. Käsige Tuberkulose der trachealen und bronchialen Lymphknoten. Tuberkeln in Milz, Leber, Niere.

6. K. K., $1 \frac{1}{4}$ Jahre alter Knabe.

Klinische Diagnose: Bronchitis. Bronchopneumonie (tuberculosa ?), Enteritis, Varicellen. Zuletzt meningitische Erscheinungen. Klinischer Befund: Rechts hinten oben Dämpfung und Bronchialatmen. Rasselgeräusche. Pirquet 11 Tage ante exitum negativ. (War auch kurz nach der Aufnahme 47 Tage ante exitum schon negativ.)

Sektionsbefund: Miliare tuberkulöse Aussaat in den Lungen. Im rechten Oberlappen tuberkulöse käsige Bronchopneumonie und tuberkulöse Kavernen. Intimatuberkel der rechten Vena pulmonalis. Im rechten Unter- und Mittellappen frische tuberkulöse Bronchitis und Peribronchitis, desgleichen im linken Ober- und Unterlappen. Miliare tuberkulöse Aussaat in Milz und Leber. Käsige Tuberkulose der trachealen und bronchialen Lymphknoten, rechts mit Durchbruch in die Kaverne der Lunge. Tuberkulose der Tonsillen und retroperitonealen Lymphknoten. Ältere tuberkulöse Herde der linken Niere, vereinzelte tuberkulöse Knötchen in beiden Nieren. Tuberkulose der Thyreoidea. Frische miliare Tuberkulose der Pia mater an der Hirnbasis. Hydrocephalus in- und externus.

7. E. M., $6{ }^{3} / 4$ Jahre altes Mädchen.

Klinische Diagnose: Meningitis tuberculosa. Lumbalpunktion. Tuberkelbazillen negativ. Pirquet 4 Tage ante exitum negativ.

Sektionsbefund: Allgemeine miliare Tuberkulose. Miliare Tuberkulose der Lungen, Milz, Niere, Leber, Nebennieren, Pankreas, Peritoneum. Käsige Tuberkulose der bronchialen Lymphknoten rechts und der Bifurkationslymphknoten. Tuberkulöse Zerebrospinalmeningitis. 
8. H. A., 4 Monate alter Knabe.

Klinische Diagnose: Meningitis tuberculosa. Bei der Aufnahme stark benommen, allgemeine Krämpfe, besonders starke Spasmen an den Extremitäten. Lumbalpunktat enthält keine Tuberkelbazillen. Pirquet 2 Tage ante exitum negativ.

Sektionsbefund: Alte käsige Bronchialdrüsentuberkulose. Tuberkulöse Bronchitis und Peribronchitis beider Lungen. Frische Miliartuberkulose der Lungen, Milz, Leber. Tuberkulöse Meningitis.

Auch hier wieder die bekannte Tatsache, dass die P i r quetsche Reaktion bei Kachektischen und schwer Tuberkulösen (besonders Meningitis tuberculosa) negativ ausfällt infolge der Reaktionsunfähigkeit des durch die Krankheit so schwer geschädigten Körpers.

Wie unbeständig aber dieser negative Ausfall der Pirquetschen Reaktion bei schwerer Tuberkulose, auch Meningitis tuberculosa und Miliartuberkulose ist, dafür mögen einige Beispiele mit positiver Reaktion trotz der schweren Veränderungen dienen.

1. E. B., 1/4 Jahre alter Knabe in äusserst schlechtem Ernährungszustand.

Klinische Diagnose: Miliartuberkulose. In ausgehebertem Mageninhalt Tuberkelbazillen. Pirquet 2 Tage ante exitum positiv.

Sektionsbefund: Miliare Tuberkulose beider Lungen mit käsiger Pneumonie im rechten Unterlappen, Kaverne im rechten Oberlappen. Miliare Tuberkulose der Pleura costalis, pulmonalis, diaphragmatica, der bronchialen Lymphdrüsen, des Dünndarmes, der mesenterialen Lymphknoten, Leber, Milz, Nieren, Pia. Durchbruch der käsigen Massen an verschiedenen Stellen in die Bronchien. In einer Vene des linken Oberlappens ein Intimatuberkel.

2. A. Z., 11/2 Jahre altes Mädchen in schlechtem Ernährungszustand (pastöser Habitus). Vater Iungenkrank.

Klinische Diagnose: Meningitis tuberculosa. Klinischer Befund: Dauernd benommen. Am Tage der Aufnahme Krämpfe. Im Lumbalpunktat keine Tuberkelbazillen. Pirquet 7 Tage ante exitum positiv.

Sektionsbefund: Meningitis tuberculosa basilaris. Konglomeraltuberkel in beiden Hemisphären und Thalamus opticus. Hydrocephalus externus. Verkäste tracheobronchiale Lymphdrüsen.

3. R. Sch., 10 Jahre alter Knabe.

Klinische Diagnose: Miliartuberkulose, Meningitis tuberculosa. Pirquet 1 Tag ante exitum positiv.

Sektionsbefund: Meningitis tuberculosa, verkäste 'Tuberkulose der bronchialen Lymphknoten. Tuberkulös-käsiger Herd im linken Oberlappen. Miliare Tuberkulose der Lungen, Milz, Leber, trache- 
alen und zervikalen Lymphdrüsen. Frische Miliartuberkulose der linken Pleura. Konglomerattuberkel des Kleinhirns.

4. O. W., $4 \frac{1}{2}$ Jahre alter Knabe in schlechtem Ernährungszustand.

Klinische Diagnose: Meningitis tuberculosa. Pirquet 7 Tage ante exitum positiv.

Sektionsbefund: Miliartuberkulose beider Lungen, Nieren, Milz, Leber. Meningitis tuberculosa. Verkäste Lymphdrüsentuberkulose. Tuberkulöse Darmgeschwüre, auch im Appendix.

5. G. S., 11/2 Jahre altes, grazil gebautes Mädchen.

Klinische Diagnose: Meningitis tuberculosa. Klinischer Befund: Nackensteife. Im Lumbalpunktat keine Bakterien. Pirquet 3 Tage ante exitum positiv.

Sektionsbefund: Ältere tuberkulöse Peribronchitiden in den Oberlappen, frischere in den Unterlappen. Verkäsende tuberkulöse Pneumonie mit Kavernenbildung im rechten Mittellappen. Tuberkulose der bronchialen Lymphknoten, Bronchitis, tuberkulöse Bronchitis links, miliare Tuberkulose in Leber, Milz, Nieren. Tuberkulose des Peritoneums, besonders über Leber und Milz. Tuberkulose der Tuben- und Uterusschleimhaut. Meningitis tuberculosa, Tuberkel im Ependym.

Diese Unbeständigkeit der Pirquetschen Reaktion bei schwerer Tuberkulose hat mich veranlasst, ein Kind mit Lungentuberkulose öfters zu impfen, nachdem es nach $2^{1} / 2$ monatlicher klinischer Beobachtung die Erscheinung von Meningitis und Miliartuberkulose zeigte.

K. Sch., $91 / 3$ Jahre altes, schlecht ernährtes Kind (Mutter lungenkrank). Bei der Aufnahme am 7. III. 1911 Schallverkürzung über der rechten Spitze, daselbst verschärftes Atemgeräusch, im Exspirium bronchial. Über der rechten Lunge knackende Geräusche. Am 9. III. 1911 Pirquet positiv, 11. III. im ausgeheberten Mageninhalt Tuberkelbazillen. 24. III. langsame Zunahme des Körpergewichtes, Lungenbefund bis auf rechts vorn ziemlich normal, 17. IV. 1911: Kind hustet mehr, 27. V. 1911 Befinden wechselnd, in letzter Zeit auffallend matt geworden, rechts hinten oben Dämpfung bis zum 4. Brustwirbel, vorn rechts über der Spitze Schallverkürzung. Atemgeräusch rechts über der Spitze sehr scharf, Exspirium hauchend, vorn verlängert, über der rechten Seite knackende Geräusche. 29. V. 1911: starke Kopfschmerzen, Schmerzhaftigkeit beim Beugen des Kopfes. Kernig positiv. Hat mehrmals erbrochen, nimmt sehr wenig zu sich. 7. VI. Wiederimpfung. 
8. VI. 1911 Pirquet negativ. 9. VI. ermeute Impfung. Somnolenz nimmt zu, reagiert fast nicht mehr, Lähmung des Musculus rectus externus oculi dextri, Puls geht langsam in die Höhe.

10. VI. Pirquet negativ, Abduzenslähmung rechts, ausgeprägter, Temperatur morgens $39^{\circ}$, Spinalpunktion: unter starkem Druck entleert sich klare Flüssigkeit, welche sich beim Stehenlassen trübt.

11. VI. wieder normale Temperatur, Allgemeinbefinden bedeutend besser. Pirquet vom 9. VI. positiv, desgleichen vom 7. VI.

12. VI. erneuter Anstieg von Puls, Temperatur und Atmung, beide Pirquet noch schwach positiv, orneute Impfung.

13. VI. Temperatur dauernd um 39, Puls und Atmung steigen noch mehr an, die Reaktionen der ersten beiden Impfstellen erlöschen, dritte Impfung negativ.

14. VI. Exitus.

Sektionsbericht: Ältere kavernöse Phthise des rechten Ober- und Unterlappens, frischere der linken Spitze. Tuberkulöse Bronchitis, Peribronchitis und Bronchopneumonie im linken Oberlappen und rechten Mittel- und Unterlappen, ältere Verwachsungen der ganzen rechten Pleurablätter, frische Tuberkulose der linken Pleura, verkäste Tuberkulose der trachealen, bronchialen und mesenterialen Lymphknoten. Tuberkulöse Leptomeningitis mit Verkäsung an einzelnen Stellen, Tuberkulose des Ventrikelependyms, mässiger Hydrocephalus internus, käsige Tuberkulose des Peritoneums parietale und viscerale, Verwachsungen des Darms, der Nieren, Leber, Milz und des Genitalapparates. Tuberkulöse Geschwüre im Dünndarm, Tuberkulose der Nieren, käsige Oophoritis, Salpingitis, Endometritis.

Man sieht also aus diesem Fall, dass bei mehrmaliger Impfung, bei schwerer Miliartuberkulose und Meningitis tuberculosa der Ausfall der Pirquetschen Reaktion wechselt. Es ist geradezu auffallend, dass dieser so schwer geschädigte Körper an dem einzigen Tage, an dem sich das Allgemeinbefinden scheinbar gebessert hatte, wieder die Kraft fand, auf die Tuberkulinimpfung zu reagienen.

Diese zahlreichen Fälle von klinisch sicherer Tuberkulose mit teils positivem, teils negativem Pirquet lassen uns wohl mit Recht folgender Schluss ziehen: Bei akuter miliarer Aussaat bekommen wir positive Reaktion, wenn auch geringfügige ältere, tubarkulöse Herde vorhanden sind, während dieselbe bei einer schon längere Zeit bestehenden tuberkulösen Erkrankung mit schweren Veränderungen mit der Zeit negativ wird. Massgebend ist die jeweilige Fähigkeit des Körpers, noch auf Tuberkulin zu reagieren oder nicht. (Vergleiche Veit!) 
Nicht uninteressant dürfte es sein, die tuberkulöse Belastung (direkte Verwandte und ständige Umgebung der Kinder) mit dem Befund der Pirquetschen Reaktion zu vergleichen.

$128 \mathrm{mal}$ war eine tuberkulöse Belastung zu konstatieren $==16 \%$.

78 mal war auch der Pirquet positiv $=60,9 \%$ der Belasteten.

50 mal war die Pir quet sche Reaktion trotz Belastung regativ $=39,1 \%$ der Belasteten, so dass sich folgende Verhältnisse ergeben :

I. Klinisch sicher tuberkulöse Fälle.

Unter den 40 Fällen waren:

21 mit positivem Pirquet und positiver Belastung $=52,5 \%$,

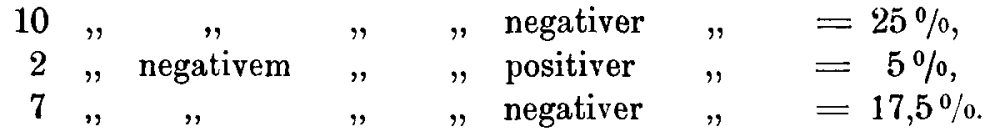

II. Klinischnichtsicher tuberkulöse Fälle.

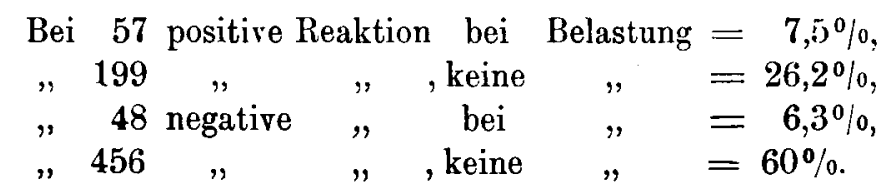

Man sieht also, dass die Pirquetsche Reaktion bei Belasteten häufiger positiv als negativ ausfällt, ferner, dass die positive Reaktion bedeutend häufiger bei sicher Tuberkulösen mit Belastung ist, als die positive Reaktion bei nicht sicher Tuberkulösen mit und ohne Belastung.

Diese Arbeit bestätigt die auch schon von anderen Autoren, Pirquet, Feer, Veit usw. gefundene Tatsache, dass der Ausfall der Pirquetschen Reaktion in den ersten drei Lebensjahren, besonders aber im Säuglingsalter als vollwertiges, sicheres diagnostisches Hilfsmittel der Diagnose der Tuberkulose des Kindesalters angesehen werden darf. In den höheren Lebensjahren lässt sich der Ausfall der Pirquetschen Reaktion nicht so verwerten wegen des Überwiegens der inaktiven Tuberkulose. Hingegen ist der negative Ausfall der Reaktion bei klinisch sicher Tuberkulösen stets als ungünstiges prognostisches Zeichen zu betrachten.

Bezüglich der Verbreitung der Tuberkulose unter der ärmeren Bevölkerung scheint mir aus den von mir beigebrachten Zahlen hervorzugehen, dass in Freiburg die Häufigkeit der Tuberkulose ungefähr in der Mitte steht zwischen der einer Grossstadt wie Wien und der einer industrielosen gut situierten Kleinstadt wie Zille. 


\section{Literatur.}

1. Feer, A., Die kutane Tuberkalinprobe ( med. Wochenschr. 1908. Nr. 1.

2. Derselbe, Uber den Wert der kutanen und konjunktivalen Tuberkulinprobe beim Kinde und über das Wesen der Skrofulose. Beitr. z. Klinik d. Tuberkulose.

3. Gangh of er, Wiener klin. Wochenschr. 1909. Nr. 4.

4. H a m bu rge r, F., Allgemeine Pathologie und Diagnostik der Kindertuberkulose. Wien 1910. F. Deuticke.

5. Hillenberg, Zur Entstehıng and Verbreitung der Tuberkulose. Zeitschr. f. Hygiene, Bd. 64, S. 306.

6. Le nhartz, Ärztl. Verein in Hamburg. 29. Oktober 1907.

7. Morgenroth, Die kutane Tuberkulinimpfung nach v. Pirquet bei Kindern des 1. Lebensjahres. Münchn. med. Wochenschr. 1908. Nr. 26.

8. v. Pirquet, Berliner med. Gesellschaft 8. und 15. Mai 1907.

9. Derselbe, Die Allergieprobe zur Diagnose der Tuberkulose im Kindesalter. Wiener med. Wochenschr. 1907. Nr. 28.

10. Derselbe, Der diagnostische Wert der kutanen Tuberkulinreaktion bei der Tuberkulose des Kindesalters, auf Grund von 100 Sektionen. Wiener klin. Wochenschr. 1907. Nr. 38.

11. Veit, Über die Ergebnisse der Kutanreaktion nach v. Pirquet. Aus dem allgemeinen Krankenhaus Hamburg-Eppendorf 1909.

12. Radziejewski, Über kutane Tuberkulinreaktion an der Universitätskinderklinik Breslau. Zeitschr. f. Kinderheilkunde 1911. 